\title{
CONTEMPORARY ASTRONOMY IN IRAN - A STATUS REPORT
}

\author{
Y.SOBOUTI \\ Institute for Advanced Studies in Basic Sciences \\ P.O.Box: 45195-159, Zanjan, Iran
}

There are of the order of 30 astronomers with research records and another 40-50 with substantial education in astronomy and astrophysics. Geographically, astronomical and astrophysical research is concentrated mainly at Shiraz University (cosmology and photometric observations), Sharif University of Theran (cosmology and gamma-ray astronomy), Tabriz University (binaries and solar physics), Meshad University (binaries and interstellar matter), Zanjan University (stellar dynamics, radio astronomy) and the Institute for Advanced Studies in Basic Sciences, Zanjan (stellar and stellar systems studies).

Observationel facilities are:

One $51 \mathrm{~cm}$ cassegrain, a number of smaller telescopes, and a photometric and CCD cameras at Biruni observatoryShiraz; One $60 \mathrm{~cm}$, one $40 \mathrm{~cm}$ cassegrain, one $15 \mathrm{~cm}$ solar telescope, filters and photographic accessories at Tabriz University; One C14 celestron and CCD at Meshad University; One C14 celestron, CCD camera and photometers at the Institute for Advanced Studies, Zanjan; Two small radio dishes at Zanjan University. Some gamma-ray facilities are being created at Sharif University.

The research record of the astronomical community of Iran is best summarised by saying that of the order of 15 papers based on research in Iran find their way to international journals and twice as many get published in internal journals. These figures are growing at a healthy rate.

The Astronomical Society of Iran came into its formal existence in 1996. Before this date it was functioning as one of the committes of the Physical Society of Iran. The Society sponsors annual meetings of astronomers and organizes regular schools at two elementary and advanced levels for young astronomers. It has a membership of 300 , including student membership. The Astronomical Society of Iran is now carryig out a feasibility study for setting up a national observatory to be available to all astronomers in the country and to guests from other places. There are numerous accessible locations in Iran with good seeings and more than 250 clear nights a year. The science Research Council of Iran sponsors this study.

The Institute for Advanced Studies in Basic Sciences of Zanjan has, by far, the most extensive international links, as regards participations in scientific meetings and other collaborations. The 23rd International School for Young Astronomers was also held in Zanjan in July 1997.

The B.Sc. curriculum of physics in Iranian universities includes astronomy and astrophysics as options. At least ten physics departments offer such options. Astrophysics at M.Sc. and Ph.D. levels is also done within the physics departments. Only recently a "Center for Astronomical Research" has been established in Tabriz University. Of the order of 10 graduate students per year choose astronomical and astrophysical problems as their thesis topics.

History of astronomy, particulary that of the Persian and Islamic astronomers of medieval and earlier times is being pursued by a number of very capable scholars. Several monograms and scholary articles have been published in this context in recent years. 
Amateur astronomers clubs are numerous and are distributed throughout the country. They draw their membership from all wlaks of life. The Municipality of Isfahan has taken upon itself to promote public awareness of astronomy and spends substantial sums to run a permanent astronomical exhibition and a planetarium. Since eight years ago a very respectable monthly, "Nojum" (meaning astronomy), is being published. It has a wide readership among the university and high school students and teachers, as well as among the immense number of amateur astronomers. 